\title{
Inclusión Social/Educativa, en Clave de Educación Superior
}

\section{Social/Educational Inclusion, in the Key of Higher Education}

\author{
Sonia Brito ${ }^{1 *}$ \\ Lorena Basualto Porra ${ }^{2}$ \\ Luis Reyes Ochoa ${ }^{2}$ \\ ${ }^{1}$ Universidad Autónoma de Chile, Chile \\ ${ }^{2}$ Universidad Católica Silva Henríquez, Chile
}

\begin{abstract}
El artículo tiene como propósito problematizar la inclusión social y educativa, en clave de educación superior. Se parte del presupuesto que la educación es un quehacer esencial e irrenunciable del progreso de la humanidad que posibilita el desarrollo de las personas y potencia sus capacidades en aspectos morales, sociales y culturales. Se aborda el tema de la inclusión desde el desarrollo del binomio social y educativo, como una forma de enfrentar la educación desde una arista social, donde ella es considerada como un derecho humano, por tanto, intrínsecamente inclusiva. El abordaje de la temática se realizará desde una metodología bibliográfica documental, que considera como fuentes principales las orientaciones de la UNESCO, especialmente, la Declaración de Salamanca y el marco de acción para las necesidades educativas especiales (UNESCO,1994), la política pública nacional y algunos autores que tratan críticamente el tema; desde esta revisión, se propone abordar la inclusión en la educación superior, desde una dimensión integral, expresada con el concepto social/educativa. De esta manera, el artículo propone una definición tipológica de inclusión que comprende los conceptos de pertenencia, igualdad, equidad y justicia social, dimensiones que debieran estar presentes en las políticas educativas de lo contrario, la inclusión continuará, paradojalmente, siendo excluyente.
\end{abstract}

Descriptores: Educación superior; Desigualdad social; Derecho a la educación; Bienestar del estudiante; Integración social.

The purpose of the article is to problematize social and educational inclusion, in the key of higher education. It assumes that education is an essential and inalienable task of the progress of humanity that enables the development of people and enhances their abilities in moral, social and cultural aspects. The issue of inclusion is approached from the development of the social and educational binomial, as a way of facing education from a social edge, where it is considered as a human right, therefore, intrinsically inclusive. The approach to the subject will be carried out from a documentary bibliographic methodology, which considers UNESCO's guidelines as main sources, especially the Salamanca Statement and the framework for action for special educational needs (UNESCO, 1994), national public policy and Some authors who critically deal with the subject; from this review, it is proposed to address the inclusion in higher education, from an integral dimension, expressed with the social/ educational concept. Thus, the article proposes a typological definition of inclusion that includes the concepts of belonging, equality, equity and social justice, dimensions that should be present in educational policies otherwise, inclusion will continue, paradoxically, being exclusive.

Keywords: University education; Social inequality; Right to education; Student welfare; Social integration.

*Contacto: sonia.brito@uautonoma.cl

ISSN: 0718-7378

www.rinace.net/rlei/
Recibido: 20/08/2019

$1^{\text {a }}$ Evaluación: 30/08/2019

Aceptado: 25/09/2019 


\section{Introducción}

La educación, presenta distintas dificultades en diversas áreas, tales como la política pública, la economía, la gestión, la ciencia pedagógica, entre otras. Ahora bien, el tema de la inclusión en la educación superior suscita gran interés, puesto que la universidad es un lugar, donde se suele replicar/amplificar las segregaciones y desigualdades sociales que operan en otros ámbitos sociales.

La educación inclusiva, es considerada un movimiento cuyo propósito es ampliar la mirada respecto del sistema educativo, reconociendo el derecho innegable a la educación y el valor de la diversidad de los estudiantes. Este derecho humano universal, lo recoge la Declaración de Salamanca (UNESCO, 1994), quien a principio de la década de los noventa manifiesta la necesidad de levantar políticas integradoras para jóvenes y adultos, en enseñanza secundaria y superior.

A pesar de esos acuerdos internacionales, en la actualidad aún existen niños, niñas y jóvenes que estar privados de ese derecho humano fundamental (UNESCO, 2005). En la misma línea argumental, la Declaración Universal de los Derechos Humanos señala, que el propósito de la educación es lograr el conocimiento y el desarrollo de todas las personas. La educación entonces debe ser inclusiva, fortaleciendo la reciprocidad y propiciando la paz (UNESCO, 2010). Por lo tanto, una educación de tipo inclusiva debe instar al desarrollo equitativo considerando las distintas visiones y miradas para su aplicación.

La amplitud del concepto de inclusión permite vincular la educación con aspectos sociales, culturales y políticos, que, en su conjunto, permiten el acceso, permanencia y resultados favorables de todas y todos los jóvenes, siendo prioritarios aquellos que se encuentran en riesgo o vulnerados en sus derechos (UNESCO, 2005). Desde esta perspectiva, "la inclusión representa un impulso fundamental para avanzar hacia la Educación para Todos, por lo que ha de concebirse como una política del ministerio de educación en su conjunto y de otros ministerios como los de salud, bienestar social o economía” (UNESCO, 2007, p. 39).

El propósito de la educación inclusiva es velar por un acceso universal de niños, niñas y jóvenes, a este sistema, potenciando lo estatal, pero también significa incorporar el componente de diversidad que agrega valor a las relaciones e intercambio y encuentro entre realidades y contextos. UNESCO (2007) manifiesta que, "Una de las finalidades de la inclusión es enfrentar la exclusión y segmentación social, por lo que una de sus principales señas de identidad es el acceso a escuelas plurales, que son el fundamento para avanzar hacia sociedades más inclusivas y democráticas" (p. 39). Esta perspectiva de reconocer lo diverso, instala desafíos al estado y a la sociedad, en tanto establecimiento de otras políticas como dispositivos que respondan de mejor manera a la construcción de prácticas y políticas inclusivas, como señala UNESCO (1994) es necesario que en el plano educacional se instalen políticas educativas integradoras.

Progresar en esta misión, es una labor de todos los sectores y actores sociales como señala UNESCO (1994), junto con el Ministerio de educación y las escuelas, en este propósito están involucrados los distintos actores sociales tales como; las familias, las organizaciones de voluntarios, las universidades, las empresas, los organismos internacionales, entre otros.

Para aportar en este empeño, OREALC/UNESCO (2007) articula asesorías técnicas, realiza promoción de políticas, articula prácticas inclusivas, desarrolla difusiones de publicación, investigaciones, evaluaciones y capacitaciones con el propósito de reunir datos e 
información pertinente respecto de la educación inclusiva de la región. Estas acciones favorecen cambios en las concepciones, y prácticas de los distintos actores relacionados con la educación, aportando al despliegue de una agenda de educación para todos y todas. El traducir este derecho a prácticas concretas de ejercicio de igualdad, requiere de soportes estatales "que garantice el acceso, la permanencia, la participación y los logros de todos los estudiantes, con especial énfasis en aquellos que, por diferentes razones, están excluidos o en riesgo de ser marginados" (UNESCO, 2009, p. 9).

Lo anterior, exige visibilizar y valorizar los dispositivos, mecanismos y esfuerzos intelectuales realizados por diversas entidades y documentos programáticos. Algunos de ellos han sido señalados en la presentación del encuentro de Ginebra (UNESCO, 2008), donde se destaca el camino recorrido en la esfera de la inclusión. Se inicia, con la misma Declaración universal de los derechos humanos (ONU, 1948), pasando por hitos importantes como la Declaración de los derechos del niño (UNICEF, 1989), la Declaración Mundial sobre Educación para Todos (UNESCO, 1990), la Declaración de Salamanca (UNESCO, 1994), el Marco de acción de Dakar (2000), Educación para todos: Un asunto de derechos humanos (UNESCO, 2007), Conferencia de Ginebra (UNESCO, 2008); y otros más actuales como Invertir en la diversidad cultural y el diálogo Intercultural (UNESCO, 2009), Compendio mundial de la educación UNESCO (2010) y el Informe para dar seguimiento a la Educación en el Mundo (2017). Otros organismos han hecho esfuerzos al respecto como la UNICEF (2000), Ciclo de Debates: Desafíos de la Política Educacional; otros esfuerzos regionales como el documento del PNUD (2013), Informe sobre Desarrollo Humano; y otros más locales como la OCDE (2009), OCDE (2013) y MINEDUC (2017) relativas a la calidad e inclusión en la Educación Superior en Chile.

Junto a estos organismos, existen diversos investigadores, tales como, Booth y Ainscow, (2002), Díaz y van Grinkel (2006), Blanco (2009), Pedroza y Villalobos (2009), Rodríguez (1998), Rawls (2004), Díaz-Romero (2006), por nombrar algunos, que están preocupados por visibilizar y proponer metodologías con alcances éticos, económicos y sociales de la inclusión.

Para estas instituciones y autores, un sistema de educación inclusivo o integral debe proveer comprensiones de los derechos humanos y dispositivos para resguardarlos. Igualmente, debe procurar el perfeccionamiento en las personas en cuanto a las aptitudes para instaurar, preservar y ejercer los derechos humanos en la vida cotidiana.

No obstante, los discursos e intenciones, el informe sobre la Educación para Todos en el Mundo, (UNESCO, 2010), señala que aún existen 72 millones de niños que no acceden a la educación formal, siendo incipientes los esfuerzos por disminuir las brechas educativas. El promedio de escolarización en algunos países subdesarrollados es de 4 años, mientras que en otros más desarrollados es de 15 o 16 años. En otros países, ha mejorado levemente la tasa de matrícula en primaria; sin embargo, la brecha de desigualdad aumenta entre países ricos y pobres, respecto al promedio de años de escolarización. Son millones los jóvenes que egresan de la escuela sin haber adquirido la capacitación necesaria para integrarse con éxito en la población activa y, además, a uno de cada seis adultos del mundo se le impugna el derecho a aprender a leer y escribir.

La UNESCO (2007), en este sentido, confirma que "es necesario revisar los esquemas actuales de financiamiento de la educación para desarrollar los cambios necesarios para que el Estado cumpla su rol de garante del derecho de todos a una educación de calidad" (p. 11). En el entendido que los recursos estatales no responden eficientemente a los 
requerimientos de la nación en materia de educación, máxime cuando se exige que ésta incorpore dimensiones como calidad, equidad, en contextos de diversidad cultural y social. Es por este motivo que el artículo propone abordar el tema de la inclusión en la educación superior desde el desarrollo del binomio social y educativo. De esta manera, la educación terciaria no puede considera la inclusión sólo desde lo educativo, es decir, abordar temas como el ingreso, la permanencia, currículum, metodologías y evaluaciones, sin considerar la dimensión social, como el contexto cultural, lenguaje, familia, necesidades especiales, situación socioeconómica, perspectiva de género, entre otros.

Por tanto, se propone abordar la inclusión en la educación superior de una manera integral, donde la inclusión refiera tanto a necesidades educativas especiales, (UNESCO, 1994), como también a una educación para todos en perspectiva de derechos humanos (UNESCO, 2007). En ambos casos, los actores sociales desde la familia, el estado y los organismos internacionales son actores relevantes del proceso.

Para abordar estas temáticas, el artículo se ordena en tres apartados, en primer lugar se problematizará el concepto de inclusión en la educación superior en cuanto al reciente debate epistemológico y metodológico; en el segundo acápite se presentará la complejidad de la política pública y los mecanismos de inclusión que se han propuesto los establecimientos de educación superior en el contex to de la realidad nacional y, finalmente, se comparten algunos planteamientos con respecto a una propuesta de educación inclusiva social/educativa que se deriva de la Ley de educación superior.

\section{Delimitación problemática inclusión social/educativa en clave de educación superior}

La inclusión educativa en educación superior es un campo de estudio reciente, por tanto, su delimitación, es materia de debate epistemológico y metodológico. Desde lo epistemológico se requiere situar la inclusión educativa en otros campos semánticos, rescatándolo de los análisis parametrales; y, desde lo metodológico, el esfuerzo es proporcionar un sustento de rigor científico a los análisis y prácticas en esta materia.

De esta manera, si se revisa la bibliografía existente sobre el tema, la inclusión educativa es concebida por la UNESCO (2005), como "un proceso orientado a responder a la diversidad de las necesidades de todos los alumnos incrementando su participación en el aprendizaje, las culturas y las comunidades, reduciendo la exclusión en y desde la educación" (p. 13). Desde el enfoque de Booth y Ainscow (2002), significa "crear culturas inclusivas, elaborar políticas inclusivas y desarrollar prácticas inclusivas” (p. 16).

Desarrollar el tema de la inclusión, desde estas perspectivas, implica abordar el tema desde un enfoque integral, considerando las necesidades de todos y todo el estudiantado, bajo una dimensión cultural y comunitaria. En este sentido, desde la educación superior, el crear culturas inclusivas, requiere necesariamente reformar la universidad de acuerdo con nuevos cánones, donde se reconocen las desigualdades de base que tributan los estudiantes. Significa, por tanto, repensar el sistema educativo comenzando por el acceso y permanencia de los estudiantes a la Educación universitaria. Además, la construcción de políticas inclusivas emplaza a las instituciones a generar dispositivos y mecanismos que respondan contextualmente a las necesidades del entorno sociocultural de los estudiantes y el reconocimiento de las diversidades culturales. 
Ahora bien, si se revisa lo que ha sucedido en el ámbito de la educación superior, un estudio sobre Educación Terciaria en Chile de la Organización para la Cooperación y el Desarrollo Económico, señala que la "educación superior chilena ya había evolucionado de un sistema de élite a un sistema masivo en un periodo relativamente corto" (OCDE, 2013, p. 28). De hecho, a nivel mundial, "la matrícula en Educación Superior ha crecido constantemente, e incluso ha llegado a duplicarse, pasando de 100 millones de estudiantes en todo el mundo en el año 2000 hasta los 207 millones en 2014" (UNESCO, 2017) En Chile, en el año 2008 la matrícula en Educación Superior alcanzaba a 819.191 estudiantes; en el año 2017, esa cifra se eleva a 1.247.7466 (MINEDUC, 2017, p. 6).

De esta forma, la educación superior ha experimentado una expansión considerable, debido a las exigencias y presiones de actores sociales que antaño, no tenían acceso. Es por este motivo que la fisonomía tanto de la educación terciaria como de los estudiantes ha ido mutando progresivamente desde los años 50, donde el ingreso a la universidad era privativo para las elites que detentaban un determinado capital cultural y social. En la actualidad, el estudiantado es más diverso y complejo, puesto que proviene de diversos sectores sociales y culturales.

En razón de lo anterior, como materia de análisis y debate, es pertinente vincular la educación superior con la inclusión tanto social como educativa, por dos razones fundamentales: el primero refiere al hecho que el joven de los sectores sociales desfavorecidos debe superar las barreras económicas y sociales para acceder a la Universidad, por las consabidas desigualdades que arrastran de la formación escolar, y segundo, debe superar las condiciones y barreras culturales para equiparar las desviaciones y desventajas de un sistema inequitativo. Lo anterior, exige plantearse la pregunta, respecto del alcance del concepto de inclusión en la educación superior, pues no puede abarcarse sólo desde el área educativa, sino que además es perentorio incluir lo social, de allí el binomio propuesto de inclusión social/educativa. El primer alcance del neologismo refiere a la inclusión social, es decir la atención a grupos considerados prioritarios, por estar viviendo en situación social desventajosa, en estado de vulneración, discriminación o inequidad. Por su parte, el alcance de la inclusión educativa refiere a una noción y una práctica, en tanto, da cuenta de un anhelo y un horizonte de reconocimiento de derechos, esto es, la consideración y valoración de los sujetos y colectividades independientes de su grupo de procedencia, bajo el prisma de la educación en diversidad. Para que esto suceda, una condición sine qua non es igualar las oportunidades, erradicar las discriminaciones y potenciar las manifestaciones de cada particularidad, siendo el eje el reconocimiento de lo diverso.

Las prácticas inclusivas, de lo que se desprende una noción de inclusión social/educativa, refiere a la traducción de los principios sustentados en el respeto, tolerancia y justicia social. Por eso, los mayores obstáculos para traducir en la práctica estos cometidos, están en directa relación con la procedencia social y cultural de las familias de los/las jóvenes; puesto que lo primero que se debe superar son las barreras económicas, culturales e instrumentales, cuyo ejemplo paradigmático es la Prueba de Selección Universitaria (PSU), para luego superar las barreras de una carrera que exige el conocimiento y apropiación de un lenguaje propio totalmente ajeno al contexto social de los estudiantes, siendo esta una de los principales caudales de abandono de los jóvenes.

El segundo alcance del neologismo refiere a lo propiamente educacional, uno de los aspectos refiere al ingreso pues, si bien el acceso a las Instituciones de Educación Superior 
se ha masificado, subsisten las desigualdades respecto de la política educativa, en cuanto las familias de los estudiantes deben pagar por la educación, aun cuando pertenezcan a los tres primeros quintiles de la población. Se puede aludir también, a las asimetrías profundas en la formación escolar, lo que redunda en que los estudiantes deban elegir universidades que tengan bajas barreras de ingreso y muchas veces escasos soportes y mecanismos para su permanencia, por tanto, el estudiante sufre una doble exclusión.

Por su parte, la OCDE (2013), señala que:

\begin{abstract}
el hecho de que los estudiantes chilenos, o sus familias, tengan que pagar directamente una parte sustancial del coste de los cursos ha centrado la atención en la relación calidad-precio de la enseñanza y en las posibilidades de encontrar una carrera profesional que merezca la pena una vez obtenida la titulación. (p. 32)
\end{abstract}

Los préstamos privados que obtienen los estudiantes y sus familias les generan un sobreendeudamiento, con altos intereses, que éstas no están en condiciones de asumir, lo que provoca cadenas de exclusiones en el acceso a la educación. Dicho de otro modo, "por cada estudiante de bajos recursos que con esfuerzos excepcionales logra acceder a la educación superior, cinco estudiantes de los sectores más acomodados ingresan a la Universidad sin mayores problemas" (OPECH, 2010, p. 3). Esta realidad también es destacada por la OCDE (2009), en su informe acerca de la educación superior en Chile, cuando señala que "los alumnos de escuelas municipalizadas que dan la Prueba de Selección Universitaria también tienen las más bajas posibilidades de aprobarla y obtener los puntajes más altos" (p. 28).

En la misma línea argumental, la OCDE (2009) agrega que:

los colegios a los que van jóvenes de familias acomodadas tienen consistentemente
mejores tasas de graduación de la educación media, preparación para la admisión a
la universidad y éxito en la universidad. Una gran cantidad de alumnos de grupos
de bajos ingresos y de escuelas municipales rinden la principal prueba de admisión
universitaria en Chile, la PSU, pero tienen peores resultados que los que podrían
predecirse de su tasa de graduación de la educación media. (p. 13)

Esta falta de igualdad y equidad en el acceso a la educación superior se explican en razón de las desigualdades multisistémicas circulares, a las divergencias educativas y las diferencias culturales que se viven en el país. Todas ellas tienen un denominador común; las brechas económicas y políticas públicas segregadoras. Cabe recordar que, según la OCDE (2018a) Chile es uno de los países con más desigualdad en el mundo, alcanzando un coeficiente de Gini de 0,47, por tanto, las políticas públicas sociales impulsados por los gobiernos de transición post dictadura, aunque se han hecho algunos avances, no han podido crear condiciones de igualdad (Urzúa, 2018).

Consecuencia de lo anterior, es el tercer alcance que se quiere presentar en cuanto que el acceso a la educación superior no asegura movilidad social, ni permanencia en las instituciones puesto que, en la actualidad, el ingreso a la educación superior no certifica entrar a los circuitos laborales remunerados acordes al esfuerzo económico y personal realizado por los estudiantes y sus familias. Para diferenciarse, las universidades, han inventado ciertas taxonomías en que se autoproclaman de excelencia, y no selectivas, por lo tanto, estos jóvenes siguen relegados a instituciones de educación superior que se autodenominan inclusivas y que operan desde la lógica de mercado y por consiguiente el estudiante responde como cliente sobre-endeudado.

Asimismo, Díaz y Van Grinkel (2006) ahondan en el tema agregando que: 
La educación no puede ser exitosa en cualquier contexto, es decir, no puede resolver por sí sola la desigualdad de los sistemas educativos y mucho menos la desigualdad en la sociedad. Además, debe ser suministrada de forma equitativa, continuada y permanente, sin ninguna discriminación social, comercial, económica o de otra índole. (p. 52)

Como señalan estos autores, el nuevo profesional cuando egresa ha superado una barrera, sin embargo, ahora debe afrontar la desigualdad social a la hora de buscar un espacio en el mundo laboral donde el capital cultural y social de los egresados es un factor determinante. De hecho, la OCDE (2018b) ha realizado un estudio donde afirma que los niños de familia con ingresos más bajos en Chile demoran seis generaciones para alcanzar un ingreso medio. A esto se agrega que, en el mercado laboral, la inequidad es ostensiblemente desventajosa para el segmento femenino con respecto a las remuneraciones y el acceso a puestos estratégicos (CASEN, 2015).

Ahora bien, como se señalaba al principio del apartado, el concepto de inclusión está en proceso de debate epistemológico, pues "la transición a la educación inclusiva no es un mero cambio técnico u organizativo, es una evolución en un sentido filosófico bien definido" (UNESCO, 2018b, p. 18). De hecho, es necesario enfrentar la paradoja que el mismo concepto de inclusión alude a discriminación, pues sugiere un sujeto excluido por otro, que ahora desea incluir. Por tanto, implícitamente, se está refiriendo a una exclusión ya sea de tipo racial, espacial, territorial, educativa, entre otras, pero que segrega y se refleja en diferentes ámbitos de la vida social y en definitiva marca una manera de estar con el otro y de convivir.

Es por este motivo que, si se esboza una definición tipológica del concepto de inclusión, es necesario, que esté presente el concepto de pertenencia, pues como señala el documento de Ginebra "Una escuela inclusiva tendrá una cultura que promueva el sentimiento de pertenencia y de interrelación y en la que cada uno sienta que se le considera como individuo" (UNESCO, 2008, p. 12).

A su vez, los conceptos de igualdad y equidad resultan fundamentales para caracterizar la inclusión, pues la educación es un derecho humano, de allí los esfuerzos de las UNESCO (1990) por instalar el concepto de una educación para todos. A su vez, la equidad, representa la igualdad en casos especiales, es por eso que "las políticas educativas deberán tener en cuenta las diferencias individuales y las distintas situaciones” (UNESCO, 1994, p. 21). En definitiva, la inclusión es justicia social que, como sostiene Rawls (2004) abre oportunidades para todos y que se caracteriza, según (Sen, 2000), por ser una experiencia social que busca la igualdad de posibilidades, con el propósito de lograr un nivel de bienestar elemental para todos los ciudadanos y ciudadanas.

\section{Políticas públicas e inclusión educativa en la educación superior}

En el primer apartado se ha problematizado sobre el concepto de inclusión en la educación superior y se ha indicado la necesidad de plantearlo desde el binomio social/educativo considerando una definición tipológica de pertenencia, igualdad, equidad y justicia social. En este segundo acápite, se presentará la complejidad de la política pública y los mecanismos de inclusión que se han propuesto para todos y todas las estudiantes de la educación superior en el contexto de la realidad nacional. Esto, porque la inclusión 
social/educativa se propone en la dinámica que señala la UNESCO (2008) de crear una cultura inclusiva lo cual, necesariamente, incluye políticas y prácticas inclusivas.

Ahora bien, a modo de diagnóstico, se observa cómo en las últimas décadas se puede visibilizar que existe una debilidad en las políticas públicas, referidas a la inclusión educativa en educación superior, puesto que el alcance de ellas refiere a políticas compensatorias que se traducen en becas (entre otras), que intentan equiparar las desviaciones de un sistema económico y social inequitativo. Por tanto, existe un discurso más bien ambiguo y escasamente comprometido con la Educación Superior. Se constata que las Universidades siguen concibiendo al estudiante universitario "heredero" de un patrimonio cultural, que conoce y está apropiado de los códigos hegemónicos, cuestión absolutamente irreal. Por tanto, los discursos, los lenguajes, como las metodologías y didácticas reproducen desviaciones y efectos paradójicos.

Las universidades han hecho eco del problema de la desigualdad y la dependencia, implementado una serie de mecanismos que buscan la inclusión. Entre ellas, han abierto la oferta de carreras, dentro de la lógica del mercado, han flexibilizado los estándares de ingreso, generando becas y ayudas institucionales, propedéuticos, tutorías, consejerías. Sin embargo, la mayor posibilidad de acceder a las instituciones de educación superior no ha logrado romper con la desigualdad ni tampoco ha permitido una mayor diversidad cultural.

Por tanto, la inclusión refiere al ejercicio del acceso universal de los jóvenes que, históricamente, no han tenido posibilidad de ingresar a la educación superior. Actualmente, esta cuestión -al parecer está resuelta- dada la gama de entidades con ofertas de lo más variopinto, no obstante, la permanencia de esos jóvenes no necesariamente está mediadas con políticas y mecanismos de nivelación que permitan comprender los nuevos códigos y lenguajes de esa instancia de formación. Es así, como un estudio realizado con estudiantes universitarios de sectores populares en Argentina, señalan que la deserción se debe muchas veces a condiciones extra académicas como el condicionamiento socioeconómico y por la incompatibilidad que resulta combinar tareas académicas, laborales y familiares. Incluso, factores como el espacio en la casa, condiciones adecuadas de espacio y silencio, afectan las tareas propias de los estudiantes universitarios (Linne, 2018).

Es así como se cataloga al joven desde la carencia, porque no es portador de las claves que el académico ha perpetuado en su imaginario, se le exige como una especie de "cultura académico-universitaria”, que "invisibiliza las desiguales trayectorias socioeducativas con las que cuenta cada estudiante al momento de ingresar al ciclo superior” (Linne, 2018, p. 139). Según Ramírez y Maturana (2018), los estudiantes necesitan hacer un proceso de aculturación donde debe "desaprender prácticas adquiridas durante su experiencia escolar para desarrollar nuevas estrategias de pensamiento, planificación y prácticas sociales que le permitan insertarse en el sistema de educación superior” (p. 156).

De esta manera, se sigue reproduciendo prácticas, estilos de enseñanza como si fueran los herederos, más aun, existe a priori una discriminación hacía estos jóvenes, al compararlos con jóvenes estudiantes de entidades públicas o con su propia formación. Resultado de ello, es la desesperanza de jóvenes que ingresan a la educación superior en busca de nuevos horizontes, pero muchas veces abandonan los estudios por bajos rendimientos y terminan arrastrando una alta deuda. Por tanto, además de perpetuar la situación de desigualdad, se suman deudas eternas y sueños incumplidos. Así lo demuestra el estudio realizado por 
Ramírez y Maturana (2018), al señalar que la deserción en primer año de los estudiantes más vulnerables alcanza un $32 \%$, cifra que está ligada con un bajo rendimiento en las notas de enseñanza media y bajo puntaje en la PSU.

La inclusión, entonces, requiere compromisos éticos tanto de las instituciones de educación superior, como del Ministerio de educación y particulares, puesto que los jóvenes y sus familias depositan confianza y esperanza de movilidad social que nunca llegan, debido a que la educación está en manos del mercado. La universalización de la Educación Superior, demanda como imperativo moral la regulación de las universidades, revisión de los planes de estudio y monitoreo permanente de los procesos de calidad y pertinencia de la gestión que evidencie las políticas y dispositivos. Un instrumento posible es la acreditación, aunque no necesariamente es efectivo, ejemplo de ello son los diversos planteles educacionales que se han cerrado en Chile por declararse en quiebra, arrastrando los sueños de tantos jóvenes estudiantes que deben luchar por reintegrarse el sistema y donde la institución no se hace responsable de lo acontecido (Brito, Basualto y Berríos, 2019).

De esta forma, el arribo a la educación superior de jóvenes "otros", requiere cambiar las claves de discusión y situar e imaginar una educación que considere las diversidades sociales que hoy ingresan a la educación superior universitaria. Lo anterior, necesita del reconocimiento que en esos espacios educativos se han experimentado transformaciones a nivel de cartografía, es decir, la emergencia de nuevos actores sociales, lo que implica un renovado arquetipo de gestión, y por tanto de instalación de propósitos con otros cánones. Por tanto, se demandan sistemas educativos que se ajusten en pertinencia a los contextos y requerimientos culturales, ideando una nueva entidad, que además de la preocupación por el saber/hacer, vuelque la preocupación por los sujetos que ingresan, los cuales necesitan apropiarse de los discursos y prácticas propias de la universidad.

El ejercer el derecho a la educación requiere necesariamente que sea inclusiva y que certifique el progreso y enseñanza para todos y todas. Esta debe plasmarse en una formación relevante, apropiada y situada, acorde a las diferencias contextuales y culturales, que aborde las metodologías de enseñanza y los ritmos de estudio. A modo de ejemplo, Linne (2018) plantea que los estudiantes de sectores populares de Argentina perciben la diferencia cualitativa de la educación superior con respecto a la educación secundaria cursada, la primera, se percibe de mayor intensidad en cuanto a la exigencia y autonomía para la cual no han sido preparados. Junto a lo anterior, ellos mismos perciben la diferencia de capital cultural con respecto a sus compañeros que han tenido la oportunidad de una mejor educación formal e informal, cuestión que se evidencia en aspectos fundamentales tales como, la lecto-escritura y el repertorio lingüístico.

La educación debe tener como sustento, la no discriminación, el acceso igualitario y la inclusión, considerando las diferencias como soporte de sociedades más equitativas y democráticas. Esta demanda social, en el caso chileno, ha sido posible debido a que los mismos jóvenes estudiantes, a través de los movimientos sociales han levantado la discusión política de la gratuidad en la educación superior, como señala Villota y Araya (2018) "estos jóvenes han sido capaces de relevar la importancia de la calidad de la educación y la tensión que generan las lógicas del mercado en este ámbito” (p. 103).

El cuestionamiento a la política de gratuidad es si, verdaderamente, ella se consolida como un dispositivo de inclusión. Al respecto es necesario señalar, que hasta el 2015 la política pública poseía un modelo subsidiario para el ingreso a la educación superior; con la 
implementación de la ley de gratuidad, el Estado considera que se trata de un derecho social. Sin embargo, se otorga solamente a algunos estudiantes que reúnen ciertas características socioeconómicas (Díaz-Romero, 2016), desde esta perspectiva, la inclusión económica es para algunos. Según el MINEDUC, (2018a) alcanza al 60\% de la población más vulnerable. Junto con lo anterior, es necesario considerar que no todos los planteles de educación superior cumplen con los requisitos para acceder a la gratuidad y algunos han decidido no implementarla debido a la incertidumbre del costo económico asociado (Littin y Rubio, 2017). Por tanto, de trata de una ley de gratuidad donde los estudiantes pueden optar a algunas universidades, de modo que no se garantiza una elección universal, lo que se hace más complejo en las zonas del país que cuentan con menos centros educativos. De esta manera, el acceso a la educación abierta, plural y universal sigue siendo privilegio de algunos estudiantes que poseen las condiciones económicas para acceder a ella. Finalizando este punto, la ley de gratuidad que no es integralmente un dispositivo de inclusión, necesita ajustarse a lo planteado por la nueva Ley de educación Superior cuando señala que: "La educación superior debe estar al alcance de todas las personas, de acuerdo a sus capacidades y méritos, sin discriminaciones arbitrarias para que puedan desarrollar sus talentos" (MINEDUC, 2018b, art. 1).

\section{Hacia una educación inclusiva integral}

Desde los apartados anteriores, se desprende que, el concepto de inclusión emerge como un imperativo ético y una necesidad de visibilizar a aquellos sectores sociales y culturales excluidos. En este sentido, se concuerda con Blanco (2009) cuando señala que:

$$
\begin{aligned}
& \text { romper el círculo vicioso de la exclusión educativa y social requiere en primer lugar } \\
& \text { visibilizar a los excluidos, porque no todos los marginados son "igualmente visibles", } \\
& \text { identificar los obstáculos que enfrentan en los ámbitos educativo y social y desarrollar } \\
& \text { políticas intersectoriales que den respuesta integral a sus necesidades. (p. 88) }
\end{aligned}
$$

En razón de esta urgencia, es que Educación 2030 (2015) propone como uno de sus objetivos para la educación del futuro "Garantizar una educación inclusiva y equitativa de calidad y promover oportunidades de aprendizaje para todos" (Objetivo 4). Al respecto, la Ley de Educación Superior incorpora la inclusión como un principio que debe regir a la educación terciaria, al respecto señala:

\section{El Sistema promoverá la inclusión de los estudiantes en las instituciones de educación superior, velando por la eliminación y prohibición de todas las formas de discriminación arbitraria. En este sentido, el Sistema promoverá la realización de ajustes razonables para permitir la inclusión de las personas con discapacidad (MINEDUC, 2018a, art. 2e)}

Como se puede apreciar, el artículo refiere al acceso a la educación superior y su obligación de incluir a todos los estudiantes eliminando discriminaciones arbitrarias, en este sentido, norma el ingreso para que todos puedan acceder, dedicando una glosa a las personas con discapacidad. Al respecto, es necesario considerar que el documento de Salamanca (UNESCO, 1994) considera para la educación terciaria la dimensión inclusiva, lo cual es ratificado por la UNESCO (2004) cuando insiste en el tema del acceso a la educación superior. Por tanto, es importante que este elemento se haya incluido, en la Ley de educación Superior chilena.

Además, señala la Ley, el principio de inclusión, tiene su fundamento en el respeto y promoción de los derechos humanos pues señala, que la "discriminación arbitraria, atentan 
contra los derechos humanos y la dignidad de las personas" (MINEDUC, 2018a, art. 2i). Como se señaló en el primer apartado, este aspecto, de contextualizar la inclusión como un derecho fundamental es propio de lo declarado por la UNESCO.

Finalmente, la Ley contempla "programas especiales de acceso, de carácter general, los que, de acuerdo con el principio de inclusión, deberán tener por objeto fomentar la equidad en el ingreso de estudiantes" (MINEDUC, 2018a, art. 13). El mismo tex to legislativo, afirma que ya existen estos tipos de planes como el Programa de Acompañamiento y Acceso Efectivo (PACE), correspondiente a políticas de inclusión (MINEDUC, 2018a, art. 81a). Cabe señalar que este programa se inicia el 2014 con un programa piloto de cinco universidades, el cual consiste, en implementar la inclusión y la equidad en los sectores más vulnerables donde los estudiantes más talentosos que, se encuentran en el $15 \%$ de mejor rendimiento de sus establecimientos, podían optar al ingreso de algunas carreras universitarias sin necesariamente alcanzar el puntaje requerido por la Prueba de selección universitaria (PSU). Además, el programa se inicia desde la enseñanza media, donde se prepara académica, vocacional y psicológicamente a los estudiantes, además de acompañarlos los primeros años de vida universitaria (Toirkens y Osses, 2016).

Lo indicado anteriormente, es lo que señala la Ley de Educación Superior del 2018 con respecto a la inclusión, no existe más explicitación del tema, por tanto, queda reducido, principalmente, a la igualdad en el acceso. Los estudios al respecto, según (Ramírez y Maturana, 2018) han relevado que, para el Ministerio de Educación, lo más importante es asegurar la inclusión desde el acceso y la permanencia, de modo que las políticas públicas se han enfocado en este ámbito, por tanto, los jóvenes beneficiados pertenecerían a un grupo socioeconómico deficitario, o bien, a jóvenes con alguna discapacidad, dejando de lado otro tipo de exclusiones.

En este sentido, la Ley de Educación Superior, invisibiliza una serie de rostros que culturalmente han sido marginados del desarrollo político, social y económico del país. Entonces, si bien hay un acento en la inclusión del acceso, queda la duda si realmente todos tienen la información y las condiciones tecnológicas y sociales para acceder a la educación terciaria. Es más, como ya se ha señalado, muchas veces la problemática no es sólo ingresar, sino mantenerse, y en este sentido la universidad también debe incluir políticas que eviten la deserción. Es por este motivo que se extraña en la ley de educación superior, una visión más integral de la inclusión que asuma otras dimensiones en el ámbito de participación, igualdad, equidad y justicia social/educativa. Es así, como en un estudio realizado por Vásquez y Alarcón (2016) sobre la inclusión en las universidades chilenas, afirman que una de las dimensiones más desarrolladas por las universidades es la admisión del alumnado, junto a otros tópicos como el respeto en el trato personal de los estudiantes, el reconocimiento de la persona y una filosofía compartida de la inclusión. Otros aspectos relacionados como ayudar a la adaptación y el acompañamiento de los estudiantes, disminuir prácticas discriminatorias, involucrar instituciones de la propia localidad, promover el trabajo en conjunto, quedan en deuda ante las políticas y culturas inclusivas promovidas por las universidades. Ahora bien, estos indicadores están en estrecha relación con el concepto de inclusión que manejan estas instituciones.

Para finalizar, según lo planteado por diferentes documentos de la UNESCO, la inclusión no puede reducirse al tema del acceso, es por este motivo que en el artículo se ha propuesto el binomio de inclusión social educativa. De allí que, las universidades deberían considerar diversas dimensiones de la inclusión, como señalan Lizama, Gil y Rahmer (2018), los 
factores que debieran estar presentes en una visión de inclusión integral, específicamente, en las universidades podría ser: desde lo social, factores socioeconómicos, la realidad familiar, las creencias y expectativas del estudiante; además de considerar los obstáculos relativos a barreras económicas, información, capital social y cultural, redes de apoyo en el mercado laboral. Por su parte, desde lo educativo, es necesario considerar la trayectoria del estudiante en cuanto a la educación preescolar, primaria y secundaria, barreras de admisión, desafíos de retención y titulación oportuna.

Es por este motivo, que una educación inclusiva integral en la educación superior es una tarea por realizarse sobre todo si se tiene en cuenta lo señalado por la UNESCO (2017), en cuanto que, el logro del desarrollo equitativo y sustentable está supeditado a los cambios de actitudes y prácticas hacia las mujeres, los migrantes, las personas con discapacidad, las minorías étnicas, religiosas, los refugiados, entre otros, es decir, los grupos históricamente excluidos. Lo anterior, requiere hacer ejercicios ciudadanos de empoderamiento, de transformaciones estructurales y de cambios profundos en la forma de entender el mundo. Considerando todos estos elementos señalados y otros emergentes, es que la educación superior necesita transitar hacia un concepto de inclusión integral, para la cual es necesario el apoyo de la política pública y la gestión institucional.

\section{Conclusión}

La educación superior ha implementado una serie de mecanismos para que los jóvenes de diversos sectores puedan ingresar a ella y, de esa manera, cumplir el sueño de ser profesionales. Sin embargo, cuando se aborda el concepto de inclusión desde una perspectiva amplia, se descubren diversas dificultades, al modo de abordar el tema por parte de las instituciones de educación superior. Así, la inclusión educativa necesita crear instancias inclusivas en la trayectoria curricular y educativa del estudiante, de lo contrario la tasa de deserción aumenta, porque no se ha podido superar la desigualdad del sistema educativo primario y secundario. Por su parte, la inserción al mundo laboral para un estudiante que no posee el capital social, cultural ni redes de contacto, es extremadamente difícil por lo que las universidades también deberían acompañar en ello y realizar un seguimiento de sus egresados.

Hasta aquí se ha referido a temáticas propias educativas, sin embargo, la propuesta del artículo es afirmar que una política inclusiva educacional en la educación superior y, eventualmente a todo nivel, no puede ser eficiente, si no se considera la dimensión social. Así, temáticas como la familia de origen, situación socioeconómica, creencias, historia, no pueden estar ajenas a la hora de proponer trayectorias educativas. A su vez, la misma vida universitaria necesita estar organizada desde una perspectiva de inclusión, propiciando en los estudiantes relacionarse con las diferentes personas y cosmovisiones que conviven en una universidad. Sin olvidar, que la institución forma parte de un contex to cultural donde la diversidad es aún mayor y que, en definitiva, será el mundo al cual está llamado a servir como profesional.

Propiciar una dinámica de inclusión educativo/social, demanda que las universidades trabajen planes de acción donde converjan todos estos elementos y así destinar recursos a una política integral de inclusión, la cual debiera estar apoyada en la política pública educativa. 
Del mismo modo, es necesario problematizar desde diversas disciplinas la categoría inclusión, puesto que, a menudo, alude a aspectos socioeconómicos, lo que permite desplegar mecanismos y dispositivos de nivelación o de acciones afirmativas ante un sistema social extremadamente inequitativo y desigual. En este sentido, su conceptualización y su práctica son remedial y jerárquica, es decir, existen aquellos que tienen el poder y el capital para incluir. La idea es ir empujando los campos semánticos para nombrar, comprender y desplegar la categoría inclusión desde la lógica del pertenecer. Lo anterior, requiere de políticas públicas sociales universales justas y prácticas culturales de equiparidad y respeto a las diversidades.

\section{Referencias}

Blanco, A. (2009). La atención educativa a la diversidad: Las escuelas inclusivas. En experiencias educativas de segunda oportunidad. Lecciones desde la práctica innovadora en América Latina. Santiago de Chile: UNESCO.

Booth, T. y Ainscow, M. (2002). Índice de inclusión: Desarrollando el aprendizaje y la participación en las escuelas. Santiago de Chile: UNESCO.

Brito, S., Basualto, L. y Berríos, A. (2019). El estado y la educación. De la industria/manufactura a Centro del saber-formador de personas. Recuperado de https://www.lemondediplomatique.cl/El-Estado-y-la-educacion-de-la.html

CASEN. (2015). Ampliando la mirada sobre la pobreza y la desigualdad. Encuesta de caracterización socioeconómica nacional. Santiago de Chile: Ministerio de Desarrollo Social.

Díaz, M. A. y Van Grinkel, H. (2006). Retos institucionales y políticos de la acreditación en el ámbito internacional. En Global University Network for Innovation. (Ed.), La educación superior en el mundo 2007. Acreditación para la garantía de la calidad: ¿Qué está en juego? (pp. 37-57). Barcelona: Mundiprensa.

Díaz-Romero, P. (2016). Gratuidad en la educación superior y su impacto en la equidad. Recuperado de http://www.uchile.cl/noticias/1 18907/gratuidad-en-la-educacion-superior-y-su-impactoen-la-equidad

Díaz-Romero, P. (2006). Caminos para la inclusión en la educación superior. Santiago de Chile: Fundación Equitas.

Linne, J. (2018). El deseo de ser primera generación universitaria. Ingreso y graduación en jóvenes de sectores populares. Revista Latinoamericana de Educación Inclusiva, 12(1), 129-147. https://doi.org/10.4067/SO7 18-73782018000100129

Littin, C. y Rubio, M. J. (2017). Educación superior: Gratuidad en el debate informativo. Santiago de Chile: Fundación superación de la pobreza.

Lizama, O., Gil, F. y Rahmer, B. (2018). La experiencia de la inclusión en la educación superior en Chile. Santiago de Chile: USACH.

MINEDUC. (2017). Educación superior e inclusión. Recuperado de http://dfi.mineduc.cl/usuarios/MECESUP/File/2017/

MINEDUC. (2018a). Ley 21091. Sobre educación superior. Recuperado de https://www.leychile.cl/N?i=1118991\&f=2018-05-29\&p=

MINEDUC. (2018b). Más de 97 mil estudiantes accedieron a gratuidad en la primera asignación 2018. Recuperado de http://www.gratuidad.cl/2018/02/20/mas-97-mil-estudiantes-accedierongratuidad-la-primera-asignacion-2018/ 
OCDE. (2009). La educación superior en Chile. Organización para la cooperación y el estudio territorial. Santiago de Chile: Ministerio de Educación.

OCDE. (2013). La educación superior en Chile el aseguramiento de la calidad en la educación superior. Santiago de Chile: Ministerio de Educación

OCDE. (2018a). Estudios económicos de la OCDE. Santiago de Chile: OCDE.

OCDE. (2018b). ¿A broken social elevator? How to promote social mobility. Santiago de Chile: OCDE.

ONU. (1948). Declaración universal de derechos humanos. Recuperado de https://www.ohchr.org/EN/UDHR/Documents/UDHR_Translations/spn.pdf

OPECH. (2010). Acceso a la educación superior: El mérito y la (re) producción de la desigualdad. Recuperado de http://www.opech.cl/inv/analisis/acceso.pdf

OREALC/UNESCO. (junio, 2007). Educación de calidad para todos: un asunto de derechos humanos. Documento de discusión en la II Reunión Intergubernamental del Proyecto Regional de Educación para América Latina y el Caribe (EPT/PRELAC). Santiago de Chile: UNESCO/OREALC.

Pedroza, R. y Villalobos, G. (2009). Políticas compensatorias para la equidad de la educación superior en Argentina, Bolivia y Venezuela. Revista de la Educación Superior, 38(152), 33-48.

PNUD. (2013). Informe sobre desarrollo humano 2013. El ascenso del sur: Progreso humano en un mundo diverso. Córdoba: PNUD.

Ramírez, L. y Maturana, J. (2018). Significado del proceso de inserción a la vida universitaria: Desde una perspectiva de aprendizaje como práctica social. Revista Latinoamericana de Educación Inclusiva, 12(1), 149-162. https://doi.org/10.4067/S0718-73782018000100149

Rawls, J. (2004). Teoría de la justicia. Ciudad de México: FCE.

Rodríguez, R. (1998). Educación y desigualdad social. Revista Mexicana de Investigación Educativa $3(6), 317-345$.

Sen, A. (2000). Desarrollo y libertad. Buenos Aires: Planeta.

Toirkens, J. y Osses, S. (2016). Estudiantes talentosos de contextos vulnerables y su ingreso a carreras de medicina. Revista Médica de Chile, 144, 534-543. https://doi.org/10.4067/So03498872016000400018

UNESCO. (1990). Declaración mundial sobre educación para todos. Jomtien: UNESCO.

UNESCO. (1994). Declaración de Salamanca y marco de acción para las necesidades educativas especiales. Salamanca: UNESCO.

UNESCO. (1998). La educación superior en el siglo XXI. Visión y Acción. París: UNESCO.

UNESCO. (2000). Marco de acción de Dakar. Educación para todos: Cumplir nuestros compromisos comunes. Dakar: UNESCO.

UNESCO. (2004). Educación superior en una sociedad mundializada. París: UNESCO.

UNESCO. (2005). Informe mundial: Hacia las sociedades del conocimiento. París: UNESCO.

UNESCO. (marzo, 2007). Educación para todos: Un asunto de derechos humanos. Documento de discusión en la II Reunión intergubernamental del proyecto regional de Educación para América Latina y el Caribe (ETP/PRELAC). Buenos Aires: UNESCO.

UNESCO. (2008). La educación inclusiva: el camino hacia el futuro. Ginebra: UNESCO.

UNESCO. (2009). Invertir la diversidad cultural y el diálogo Intercultural. París: UNESCO. 
UNESCO. (2010). Compendio mundial de la educación. Comparación de las estadísticas de educación en el mundo. Costos y compromisos asociados con el financiamiento de la educación para todos. París: UNESCO.

UNESCO. (2017). Informe para dar seguimiento a la educación en el mundo. París: UNESCO.

UNICEF. (1989). Convención sobre los derechos del niño. Recuperado de https://www.unicef.cl/web/informes/derechos_nino/convencion.pdf

UNICEF. (2000). Ciclo de Debates: Desafíos de la política educacional. Tensión entre derecho a la educación y libertad de enseñanza. París: UNICEF.

Urzúa, S. (2018). La batalla contra la desigualdad en Chile. Santiago de Chile: Libertad y Desarrollo.

Vásquez, B. y Alarcón, E. (2016). La inclusión en las universidades chilenas. Del discurso a las interacciones prácticas, políticas y culturales. Pensamiento Educativo, 53(2), 1-19. https://doi.org/10.7764/PEL.53.2.2016.9

Villota, Y. y Araya, J. (2018). Juventud, comunicación y movimientos sociales. En S. Brito y L. Basualto (Eds.), Juventudes y jóvenes. Perspectivas desde la formación integral (pp. 91-107). Santiago de Chile: UCSH.

\section{Breve CV de los autores}

\section{Sonia Brito}

Doctora en Ciencias de la Educación, mención educación intercultural por la Universidad de Santiago de Chile. Magister en Educación Superior por la Universidad Andrés Bello de Santiago Chile. Asistente Social por la Universidad Tecnológica Metropolitana. Académica en la Universidad Autónoma de Chile. Participó como investigadora principal en investigaciones acerca de los Jóvenes, la perspectiva de género, la democracia, participación social y la inclusión en la Educación Superior. Compiladora de 6 productos editoriales en formato libro, como, por ejemplo, "Formación transversal y competencias genéricas en Universidades. Clave para una renovación curricular con compromiso social". ORCID ID: https://orcid.org/OOOO-0002-1211-1125. Email:

sonia.brito@uautonoma.cl

\section{Lorena Basualto Porra}

Magíster en Teología por la Pontificia Universidad Católica de Chile. Licenciada en Ciencias Religiosas y Profesora de Religión y Moral en la Pontificia Universidad Católica de Valparaíso. Académica Adjunta en la Escuela de Ciencias Religiosas de la Facultad de Educación de la Universidad Católica Silva Henríquez. Participó en la investigación: “Jóvenes y perspectivas de género. Un estudio desde las estudiantes de la UCSH”. Es compiladora de publicaciones como "Juventud (es) y jóvenes; perspectivas desde la formación integral UCSH. Experiencias académicas desde diversos espacios formativos. Santiago: UCSH" y autora del artículo "La tensión entre una espiritualidad desde abajo y una espiritualidad desde arriba Paradojas de la Exhortación Apostólica Amoris Laetitia”. ORCID ID: https://orcid.org/0000-0001-8780-0841. Email: lbasualtop@ucsh.cl

\section{Luis Reyes Ochoa}

Doctor en Didáctica y Organización Educativa de la Universidad de Sevilla, España. Magíster en Educación mención Curriculum Educacional (UMCE). Profesor IPES Blas 
Cañas. Académico del Instituto Interdisciplinario en Pedagogía y Educación (IIPE), Facultad de Educación de la Universidad Católica Silva Henríquez. Co-autor y compilador del libro "Desafíos y tensiones en la Gestión del Currículo: Teoría y Práctica" y compilador de libros como "Docencia universitaria. Reflexión desde la práctica” y” Formación transversal y competencias genéricas en universidades. Clave para una renovación curricular con compromiso social". Cuenta con experiencia de más de 25 años en docencia e investigación en Currículum, Didáctica y Formación de Profesores en universidades públicas y privadas.

ORCID ID: https://orcid.org/o000-000 1-6070-6169. Email: Ireyeso@ucsh.cl 